\title{
GLOBAL CHARACTERIZATIONS OF THE SPHERE
}

\author{
GEORGE STAMOU
}

ABStract. Let $S$ be an ovaloid in Euclidean three-space $E^{3}$ with Gaussian curvature $K>0$ and let $K_{\mathrm{II}}$ be the curvature of the second fundamental form II of $S$. We give some global characterizations of the sphere by the curvature $K_{\mathrm{II}}$ which generalize some results of R. Schneider [4], D. Koutroufiotis [2] and the well-known "H-Satz" theorem of H. Liebmann.

Let $S$ be a closed and sufficiently smooth surface in Euclidean three-space $E^{3}$ with Gaussian curvature $K>0$, i.e. an ovaloid. By appropriate orientation the second fundamental form II defines a nondegenerate Riemannian metric on $S$. Let $H$ be the mean curvature of $S$ and $K_{\mathrm{II}}$ be the curvature of II. Then the equality

$$
K_{\mathrm{II}}=H+Q-\left(8 K^{2}\right)^{-1} \nabla_{\mathrm{II}} K
$$

holds identically on $S$, where $Q$ is a certain nonnegative function and $\nabla_{\text {II }}$ denotes the first Beltrami operator (square of the gradient) with respect to II [4]. On the closed surface $S$ there exist points $P_{0}, P_{1}$ so that

$$
K\left(P_{0}\right)=\max _{p \in S} K(P), \quad K\left(P_{1}\right)=\min _{p \in S} K(P) .
$$

Thus we have $\nabla_{\mathrm{II}} K\left(P_{0}\right)=\nabla_{\mathrm{II}} K\left(P_{1}\right)=0$, and since $Q \geqslant 0$, (1) yields $K_{\mathrm{II}}\left(P_{0}\right)$ $\geqslant H\left(P_{0}\right)$ and $K_{\mathrm{II}}\left(P_{1}\right) \geqslant H\left(P_{1}\right)$.

THEOREM 1. Let $S$ be an ovaloid in $E^{3}$. If there exists a function $f(x, y)$ which satisfies the following conditions:

(a) $f(x, y)$ is increasing in $x$ and decreasing in $y$,

(b) the function $F(x, y):=f(x, y) / x(x \neq 0)$ is decreasing in $x$, and if $K_{\mathrm{II}}=f(H, K)$ identically on $S$, then $S$ is a sphere.

Proof. Since $K_{\mathrm{II}}=f(H, K)$ and $H \geqslant \sqrt{K}$,

$$
\begin{aligned}
\frac{f(H(P), K(P))}{\sqrt{K(P)}} \geqslant \frac{f(\sqrt{K(P)}, K(P))}{\sqrt{K(P)}} \geqslant \frac{f\left(\sqrt{K\left(P_{0}\right)}, K(P)\right)}{\sqrt{K\left(P_{0}\right)}} \\
\geqslant \frac{f\left(H\left(P_{0}\right), K(P)\right)}{H\left(P_{0}\right)} \geqslant \frac{f\left(H\left(P_{0}\right), K\left(P_{0}\right)\right)}{H\left(P_{0}\right)} \geqslant 1
\end{aligned}
$$

for every $P \in S$. Then we have

Received by the editors May 23, 1977.

AMS (MOS) subject classifications (1970). Primary 53C45; Secondary 53A05, 53C40.

Key words and phrases. Ovaloid, curvature of the second fundamental form, sphere. 


$$
K_{\mathrm{II}}(P)=f(H(P), K(P)) \geqslant \sqrt{K(P)}
$$

and $S$ is a sphere (cf. [5, Corollary 5, p. 383]).

THEOREM 1'. Let $S$ be an ovaloid in $E^{3}$. If there exists a function $f(x, y)$ which satisfies the following conditions:

(a) $f(x, y)$ is increasing in $x$,

(b) the function $F(x, y):=f(x, y) / x(x \neq 0)$ is decreasing in $x$,

(c) the function $G(x, y):=f(x, y) / \sqrt{y}(y>0)$ is increasing in $y$, and if $K_{\mathrm{II}}=f(H, K)$ identically on $S$, then $S$ is a sphere.

Proof. We have

$$
\begin{aligned}
\frac{f(H(P), K(P))}{\sqrt{K(P)}} \geqslant & \frac{f(\sqrt{K(P)}, K(P))}{\sqrt{K(P)}} \geqslant \frac{f\left(\sqrt{K\left(P_{1}\right)}, K(P)\right)}{\sqrt{K(P)}} \\
& \geqslant \frac{f\left(\sqrt{K\left(P_{1}\right)}, K\left(P_{1}\right)\right)}{\sqrt{K\left(P_{1}\right)}} \geqslant \frac{f\left(H\left(P_{1}\right), K\left(P_{1}\right)\right)}{H\left(P_{1}\right)} \geqslant 1
\end{aligned}
$$

for every $P \in S$. Hence,

$$
K_{\mathrm{II}}(P)=f(H(P), K(P)) \geqslant \sqrt{K(P)}
$$

and $S$ is a sphere [5, Corollary 5, p. 383].

For appropriate choices of the constants $r$ and $s$, the function $f(x, y)=$ $c x^{s} y^{r}$, with $c>0$ constant, satisfies the hypotheses of Theorems 1 or $1^{\prime}$. For example, if $K_{\mathrm{II}}=c H^{s} K^{r}$ on $S$ with $0 \leqslant s \leqslant 1$ and $r \leqslant 0$ or $r \geqslant \frac{1}{2}$, then $S$ is a sphere. This result generalizes those of Schneider [4, Theorem 1] (for surfaces in $E^{3}$ ) and Koutroufiotis [2, Theorems 1 and 2].

LEMMA 1. Let $\bar{P}$ be a point on an ovaloid $S$ where $H$ attains its maximum. Then $K_{\mathrm{II}}(\bar{P}) \geqslant H(\bar{P})$.

Proof. According to [1, p. 7] the identity

$$
2 H\left(K_{\mathrm{II}}-H\right)\left(H^{2}-K\right)=\frac{1}{2} K \nabla_{\mathrm{II}}\left(H, H^{2} / K\right)-\frac{1}{4} \nabla\left(H^{2} / K, K\right)
$$

is valid, which easily implies

$$
\begin{aligned}
2 H\left(K_{\mathrm{II}}-H\right) & \left(H^{2}-K\right) \\
= & \frac{1}{2} K \nabla_{\mathrm{II}}\left(H, \frac{H^{2}}{K}\right)-\frac{H}{2 K} \nabla(H, K)+\frac{H^{2}}{4 K^{2}} \nabla K .
\end{aligned}
$$

Here $\nabla(\varphi, \psi)$ (resp. $\left.\nabla_{\mathrm{II}}(\varphi, \psi)\right)$ denotes the inner product of the gradients of $\varphi$ and $\psi$ with respect to I (resp. II) and $\nabla \varphi:=\nabla(\varphi, \varphi)$. The right-hand side of (2) is nonnegative at $\bar{P}$, since

$$
\nabla_{\mathrm{II}}\left(H(\bar{P}), H^{2}(\bar{P}) / K(\bar{P})\right)=\nabla(H(\bar{P}), K(\bar{P}))=0 \text { and } \nabla K(\bar{P}) \geqslant 0 \text {. }
$$

Since $H^{2}(\bar{P}) \geqslant K(\bar{P})$, the left side of (2) yields $K_{\mathrm{II}}(\bar{P}) \geqslant H(\bar{P})$. 
The following results are similar to Simon's [5, Lemma 6 and Theorem, p. 383].

LEMMA 2. Let $S$ be an ovaloid in $E^{3}$. If there exists $\bar{P} \in S$ where $K_{\mathrm{II}}$ takes its minimum and $H$ takes its maximum, then $S$ is a sphere.

Proof. We get, for every $P \in S$,

$$
K_{\mathrm{II}}(P) \geqslant K_{\mathrm{II}}(\bar{P}) \geqslant H(\bar{P}) \geqslant H(P) \geqslant \sqrt{K(P)}
$$

and the assertion follows [5, Corollary 5, p. 383].

Finally, using Lemma 2, we can prove in the same way as in [5, Theorem, p. 383]:

THEOREM 2. Let $S$ be an ovaloid in $E^{3}$. If there exists a function $\Phi(x, y)$ which is increasing (resp. decreasing) in both variables and strictly monotonic in at least one of its variables and if $\Phi\left(H(P), K_{\mathrm{II}}(P)\right)=0$ for all $P \in S$, then $S$ is a sphere.

This theorem is a common generalization of Schneider's [4, Theorem 1] (for surfaces in $E^{3}$ ) and the well-known " $H$-Satz" theorem of Liebmann (cf. [3, p. 163]).

\section{REFERENCES}

1. P. J. Erard, Über die zweite Fundamentalform von Flächen im Raum, Doctoral Thesis, Diss. No. 4234, Eidgenössische Technische Hochschule, Zürich, 1968. MR 44 \#5866.

2. D. Koutroufiotis, Two characteristic properties of the sphere, Proc. Amer. Math. Soc. 44 (1974), 176-178.

3. D. Laugwitz, Differentialgeometrie, 2nd ed., Teubner, Stuttgart, 1968.

4. R. Schneider, Closed convex hypersurfaces with second fundamental form of constant curvature, Proc. Amer. Math. Soc. 35 (1972), 230-233. MR 46 \#6254.

5. U. Simon, Characterizations of the sphere by the curvature of the second fundamental form, Proc. Amer. Math. Soc. 55 (1976), 382-384.

Department of Mathematics, Univeristy of Thessaloniki, Thessaloniki, Greece 